\title{
Effects of iron overload and exenatide on erythrocyte deformability in a rat model
}

\author{
Kuskonmaz $\mathrm{SM}^{1}$, Kara $\mathrm{H}^{2}$, Comu $\mathrm{FM}^{3}$, Kucuk A ${ }^{4}$, Arslan $\mathrm{M}^{5}$ \\ Department of Endocrinology and Metabolism Gazi University Medical Faculty, Ankara, Turkey. \\ marslan36@yahoo.com
}

\begin{abstract}
Iron overload is known to affect erythrocyte membrane properties and erythrocyte shape. We hypothesized that iron overload which directly affects the erythrocyte morphology may also interfere with erythrocyte deformability (ED). Exenatide, a glucagon like peptide -1 (GLP-1) analogue used in the treatment of diabetes, is known to have beneficial pleiotropic effects on endothelial function and blood flow which are different from its glucoselowering effects. In our study we aimed to test the effect of iron overload on ED in a rat model (1) and to evaluate the effect of exenatide on ED in the same model (2). For this purpose, the animals were randomly divided into three groups, each containing 6 rats. Rats in the control group (Group C) were given intraperitoneal injections of saline as placebo. The second group (Group Fe) was given intraperitoneal iron dextran (60 mg/kg/day) five days a week for 4 weeks to develop iron overload. The third group (Group Fe $+E$ ) received subcutaneous injections of $10 \mathrm{mcg}$ exenatide (Byetta ${ }^{\circledR}$ Lilly Pharma) in two divided doses for 4 weeks in addition to iron dextran. We observed that ED index was significantly higher in Group Fe when compared to Group $C$ and Group $\mathrm{Fe}+\mathrm{E}(\mathrm{p}<0.0001, \mathrm{p}<0.0001)$. Administration of exenatide to the iron-loaded rats (Group Fe $+E)$ led to a significant decrease in the deformability index $(p<0.017)$ when compared to Group Fe. Our study shows a decrease in ED with iron and beneficial effect of exenatide on ED in the iron-overloaded rat model (Fig. 1, Ref. 24). Text in PDF www.elis.sk. KEY WORDS: erythrocyte deformability, iron, exenatide.
\end{abstract}

\section{Introduction}

The term erythrocyte deformability (ED) represents the ability of erythrocytes to change shape and turn back to the original discoid shape (1). This important mechanical property of erythrocytes facilitates blood flow through the circulation in vessels of variable diameter and enables effective exchange of gas and metabolic products in capillaries.

Iron overload is known to affect erythrocyte membrane properties and erythrocyte shape (2). We hypothesized that iron overload which directly affects the erythrocyte morphology may also interfere with ED.

DPP-4 inhibitors (eg, vildagliptin) and GLP-1 agonists (eg, exenatide) are novel antidiabetic agents both acting through incretin system. Exenatide, a 39 amino acid peptide GLP-1 receptor

${ }^{1}$ Department of Endocrinology and Metabolism Gazi University Medical Faculty, Ankara, Turkey, ${ }^{2}$ Department of Pharmacology, Yildirim Beyazit University Medical Faculty, Ankara, Turkey, ${ }^{3}$ Department of Physiology, Kirikkale University Medical Faculty, Kirikkale, Turkey, ${ }^{4}$ Department of Physiology, Dumlupinar University Medical Faculty, Kütahya, Turkey, and ${ }^{5}$ Department of Anaesthesiology and Reanimation, Gazi University Medical Faculty, Ankara,Turkey

Address for correspondence: M. Arslan, Dr, Gazi University Medical Faculty, Department of Anesthesiology and Reanimation, 06510 Ankara, Turkey.

Phone: +90.312.2026739, Fax: +90.312.2024166 agonist, is used in the treatment of diabetes (3). It is known to have beneficial pleiotropic effects on endothelial function and blood flow (4). Vildagliptin, a DPP-4 inhibitor, is shown to improve ED in a clinical study but to date, there is no study published regarding the effect of exenatide on ED.

In this study, we aimed to investigate the effect of iron overload on erythrocyte deformability and to test exenatide effects on deformability in a rat model of iron overload.

\section{Materials and methods}

\section{Animals and experimental protocol}

In this study, 18 male Wistar Albino rats weighing between 250 and $300 \mathrm{~g}$, raised under the same environmental conditions, were used. The rats were kept under $20-21^{\circ} \mathrm{C}$, at 12-hour daylight/ darkness cycles and had free access to food until 2 hours before the anesthesia procedure. The animals were randomly divided into the three groups, each containing 6 rats. Rats in the control group (Group C) were given intraperitoneal injections of saline as placebo. The second group (Group Fe) was given intraperitoneal iron dextran (60 mg/kg/day) five days a week for 4 weeks (5) to develop an iron overload. The third group (Group Fe + E) received subcutaneous injections of $10 \mathrm{mcg}$ exenatide (Byetta ${ }^{\circledR}$ Lilly Phar$\mathrm{ma}$ ) in two divided doses for 4 weeks in addition to iron dextran.

All the rats were given ketamine $100 \mathrm{mg} \cdot \mathrm{kg}^{-1}$ intraperitoneally and intracardiac blood samples were obtained. Heparinized 


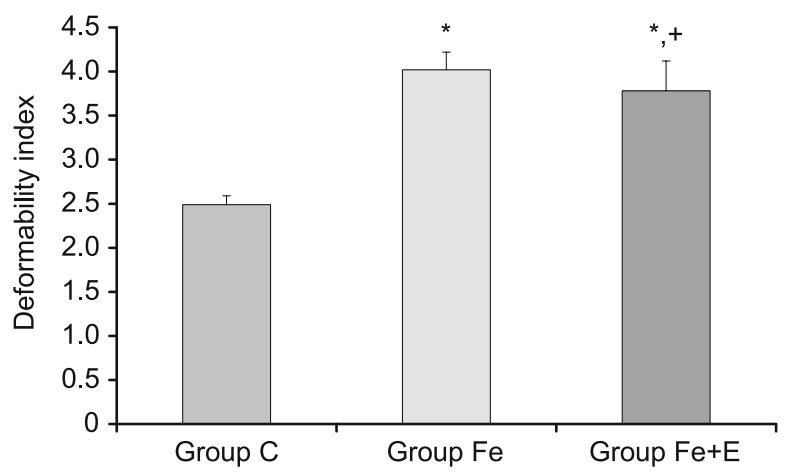

Fig. 1. Erythrocyte deformability values of the groups. Each bar represents the mean $\pm \mathbf{s d}$. * $\mathbf{p}<\mathbf{0 . 0 5}$ compared to the Group $\mathbf{C},+{ }^{*} \mathbf{p}<$ 0.05 compared to the Group Fe.

total blood samples were used to prepare erythrocyte packs. Deformability measurements were done using erythrocyte suspensions with $5 \%$ hematocrit in phosphate buffered saline (PBS) buffer.

\section{Deformability measurements}

Blood samples were taken carefully and analyzed immediately to avoid hemolysis. Collected blood was centrifuged at 1000 rpm for ten minutes. After removing the serum and buffy coat on erythrocytes, isotonic PBS buffer was added to collapse erythrocytes, and this mixture was centrifuged at $1000 \mathrm{rpm}$ for ten minutes. Liquid on the upper surface was discarded. The mixture was washed three times to obtain pure red cell packs. A suspension of $5 \%$ hematocrit was prepared by mixing the red cells with PBS buffer. The suspension was used for the measurement of deformability. Collection and deformability measurements of erythrocytes were done at $22^{\circ} \mathrm{C}$.

The constant-current filtrometer system was used for measurement of erythrocyte deformability. Samples were prepared as $10 \mathrm{ml}$ of erythrocyte suspension and PBS buffer. The flow rate was held constant at $1.5 \mathrm{ml} / \mathrm{min}$ with an infusion pump. The 28-mm nucleoporin polycabonate filter with a $5 \mu \mathrm{m}$-pore diameter was used. The pressure changes during the passage of erythrocytes through the filter were detected by a pressure transducer and the data were transferred to a computer with the help of MP 30 data equation systems (Biopac Systems Inc, Commat, USA). Pressure changes are measured various times to calculate the necessary values via computer programs. A pressure calibration of the system was performed each time before measuring the samples. First the buffer (PT) and then the erythrocytes (PE) were passed through from the filtration system and the changes in pressure were measured. The relative refractory period (Rrel) was calculated by relating the pressure value of erythrocyte suspension to that of the buffer. Increase in Rrel was interpreted as a decrease in erythrocyte deformability, while the deformability index is inversely proportional to the capability of cells to change and restore their shape $(6,7)$.

\section{Statistical analysis}

Statistical Package for the Social Sciences (version 20.0, SPSS, Chicago, IL, USA) was used for statistical analysis. Erythrocyte deformability was assessed by using Kruskal-Wallis test. Bonferroni adjusted Mann-Whitney U test was used after significant Kruskal-Wallis to determine which group differs from the other. Results were expressed as mean \pm standard deviation (Mean $\pm \mathrm{SD}$ ). Statistical significance was set at a $p$ value $<0.05$

\section{Results}

Erythrocyte deformability index was significantly higher in Group Fe when compared to Group C and Group Fe+E ( $p<$ 0.0001, $\mathrm{p}<0.0001$ ) (Fig. 1). Administration of exenatide to the iron-loaded rats (Group $\mathrm{Fe}+\mathrm{E}$ ) led to a significant decrease in the deformability index $(\mathrm{p}<0.017)$ when compared to Group Fe.

\section{Discussion}

ED is a function of red cell geometry (1), viscosity of intracellular fluid (2), and erythrocyte membrane $(3,8)$. Changes in ED are expressed by the deformability index which is inversely proportional to the capability of cells to change and restore their shape.

Iron overload is known to be associated with many pathological conditions, including liver and heart disease, neurodegenerative disorders, diabetes, and heart failure, (e.g. (9)). Iron loading is shown to increase vascular oxidative stress and interfere with the vasodilatatory response (10-12). Chronic iron overload is associated with an increased incidence of ischemic cardiovascular events, which is supposed to be related to iron-induced vascular dysfunction $(13,14)$.

Iron overload affects erythrocyte morphology. Erythrocytes lose their normal discoid shape and exhibit pointed extensions with large membrane pores (2).

Studies with electron microscopy showed various iron-induced morphological changes in erythrocytes. Lipinski et al, showed that the addition of iron to platelet-poor plasma induces formation of dense matted fibrin deposits, which is different from the typical net-like appearance of fibrin (15). The authors explained this change in morphology of fibrin by hydroxyl radical formation and suggested that ferric ions cause hydroxyl radical formation, and this in turn causes polypeptide unfolding and scrambled refolding. Similar phenomenon is also observed in hemochromatosis, a disease with natural overload (16). Red blood cells in hereditary hemochromatosis patients exhibit different membrane properties with aggregated and enlarged intramembrane particles (16). They have a greater axial ratio and a different ultrastructure (16). In this study, we demonstrated a significant decrease in ED of erythrocytes from iron-overloaded rats when compared to controls. In addition to inflammation and endothelial dysfunction, iron overload has a direct effect on ED, probably through changes induced in membrane properties. This effect may contribute to the increased incidence of cardiovascular events in disease states associated with iron overload.

GLP-1 is one of the incretin hormones which are secreted from the gut upon interaction with nutrients to regulate glucose 
disposal. GLP-1 acts through seven transmembrane G protein coupled receptors (17). The presence of GLP-1 receptors is shown in the pancreas and extrapancreatic tissues including the gut, brain, lung, kidney, and heart (18).

Studies showed an improvement in endothelial dysfunction with GLP-1 agonists $(19,20)$. Antioxidative properties of these molecules are also demonstrated in several trials $(21,22)$.

There is no reported investigation on the effect of GLP-1 agonists on erythrocytes up to date. In one clinical study, vildagliptin is shown to improve retinal blood flow and ED in diabetic patients (23). Endogenous GLP-1 is rapidly degraded by the dipeptidyl peptidase-4 (DPP-4) enzyme. Vildagliptin is a DPP-4 inhibitor developed to prolong the action of endogenous incretins and is used in the treatment of diabetes (24). However, improved glucose control in diabetics is associated with improved ED, and so is the confounding effect which precludes the assessment of direct effect of this agent - or endogenous GLP-1 promoted by this agent - on ED.

In our study we assessed the effect of exenatide on ED in an iron overload model. To our knowledge, this is the first study which tested the effect of a GLP-1 agonist on ED. We showed that exenatide provided a significant decrease in ED. This non-diabetic model prevents the confounding effect of improved glucose control on ED and supports the possibility of a direct effect of exenatide.

Further studies are needed to show the exact mechanism of action of exenatide on erythrocyte membrane properties.

\section{References}

1. Minetti G, Egee S, Morsdorf D, Steffen P, Makhro A, Achilli C et al. Red cell investigations: art and artefacts. Blood Rev 2013; 27: 91-101.

2. Pretorius E, Vermeulen N, Bester J, du Plooy JL, Gericke GS. The effect of iron overload on red blood cell morphology. Lancet 2014; 383 (9918): 722

3. Gentilella R, Bianchi C, Rossi1 A, Rotella CM. Exenatide: a review from pharmacology to clinical practice. Diabetes Obes Metab 2009; 11 : 544-556.

4. Koska J. Incretins and preservation of endothelial function. Cardiovasc Hematol Agents Med Chem 2012; 10 (4): 295-308.

5. Navidi-Shishaone M, Mohhebi S, Nematbakhsh M, Roozbehani S, Talebi A, Pezeshki $\mathbf{Z}$ et al. Co-Administration of Silymarin and Deferoxamine against Kidney, Liver and Heart Iron Deposition in Male Iron Overload Rat Model. Int J Prev Med 2014; 5 (1): 110-116.

6. Arslan M, Comu FM, Isik B, Unal Y, Cekmen N, Kurtipek O. Effects of a general anaesthetic agent, propofol, on erythrocyte deformability. Bratisl Med J 2010; 111 (3): 126-128.

7. Arslan M, Comu FM, Isik B, Oztürk L, Kesimci E. Effect of dexmedetomidine on erythrocyte deformability during ischemia-reperfusion injury of liver in diabetic rats. Bratisl Med J 2012; 113 (12): 687-691.

8. Shin S, Ku Y, Babu N, Singh M. Erythrocyte deformability and its variation in diabetes mellitus. Indian J Exp Biol 2007; 45 (1): 121-128.

9. Vujić M. Molecular basis of HFE-hemochromatosis. Front Pharmacol 2014; 5: 42. doi: 10.3389/fphar.2014.00042.
10. Day SM, Duquaine D, Mundada LV, Menon RG, Khan BV et al. Chronic iron administration increases vascular oxidative stress and accelerates arterial thrombosis. Circulation 2003; 107: 2601-2606.

11. Ahluwalia N, Genoux A, Ferrieres J, Perret B, Carayol M et al. Iron status is associated with carotid atherosclerotic plaques in middleaged adults. J Nutr 2010; 140: 812-816.

12. Depalma RG, Hayes VW, Chow BK, Shamayeva G, May PE et al. Ferritin levels, inflammatory biomarkers, and mortality in peripheral arterialdisease: a substudy of the Iron (Fe) and Atherosclerosis Study (FeAST) Trial. J Vase Surg 2010; 51: 1498-1503.

13. Kiechl S, Willeit J, Egger G, Poewe W, Oberhollenzer F. Body iron stores and the risk of carotid atherosclerosis: prospective results from the Bruneck study. Circulation 1997; 96: 3300-3307.

14. Merono T, Rosso LG, Sorroche P, Boero L, Arbelbide J et al. High risk of cardiovascular disease in iron overload patients. Eur J Clin Invest 2011; 41: 479-486.

15. Lipinski B, Pretorius E. Hydroxyl radical-modified fibrinogen as a marker of thrombosis: The role of iron. Hematology 2012; 17: 241-247.

16. Pretorius E, Bester J, Vermeulen N, Lipinski B, Gericke GS, Kell DB. Profound morphological changes in the erythrocytes and fibrin networks of patients with hemochromatosis or with hyperferritinemia, and their normalization by iron chelators and other agents. PLoS One 2014, 9 , e85271. doi: 10.1371/journal.pone.0085271. eCollection 2014.

17. Thorens B, Porret A, Buhler L, Deng SP, Morel P, Widmann C. Cloning and functional expression of the human islet GLP-1 receptor: demonstration that exendin-4 is an agonist and exendin-(9-39) an antagonist of the receptor. Diabetes 1993; 42 (11): 1678-1682.

18. Holst JJ. The physiology of glucagon-like peptide 1. Physiol Rev 2007; 87: 1409-1439.

19. Koska J, Sands M, Burciu C, D'Souza KM, Raravikar K, Liu J et al. Exenatide Protects against Glucose and Lipid-Induced Endothelial Dysfunction: Evidence for Direct Vasodilation Effect of GLP-1 Receptor Agonists in Humans. Diabetes 2015 Feb 26. pii: db140976.

20. Erdogdu Ö, Eriksson L, Nyström T, Sjöholm Å, Zhang Q. Exendin-4 restores glucolipotoxicity-induced gene expression in human coronary artery endothelial cells. Biochem Biophys Res Commun 2012; 419 (4): 790-795.

21. Sélley E, Kun S, Szijártó IA, Laczy B, Kovács T, Fülöp F et al. Exenatide induces aortic vasodilation increasing hydrogen sulphide, carbon monoxide and nitric oxide production. Cardiovasc Diabetol 2014; 1369.

22. Zhao Q, Xu CL, Xiong HY, Huang W, Zhang M, Wang Y et al. Alleviation of hyperglycemia induced vascular endothelial injury by exenatide might be related to the reduction of nitrooxidative stress. Biomed Res Int 2013; 2013: 843657. doi: 10.1155/2013/843657.

23. Berndt-Zipfel C, Michelson G, Dworak M, Mitry M, Löffler A, Pfützner A et al. Vildagliptin in addition to metformin improves retinal blood flow and erythrocyte deformability in patients with type 2 diabetes mellitus - results from an exploratory study. Cardiovasc Diabetol 2013; 12: 59 .

24. Scheen AJ. A review of gliptins for 2014. Expert Opin Pharmacother 2015; 16 (1): 43-62.

Received March 17, 2015. Accepted May 28, 2015. 